\title{
Towards a Common Framework and Database of Materials for Soft Robotics
}

\author{
Luc Marechal1, Pascale Balland1, Lukas Lindenroth2 3, Fotis Petrou4, Christos Kontovounisios4 and Fernando \\ Bello4
}

\begin{abstract}
In order to advance the field of soft robotics, a unified database of material constitutive models and experimental characterizations is of paramount importance. This will facilitate the use of finite element analysis (FEA) to simulate their behavior and optimize the design of soft-bodied robots. Samples from seventeen elastomers, namely Body Double ${ }^{\mathrm{TM}}$ SILK, Dragon Skin ${ }^{\mathrm{TM}} 10$ MEDIUM, Dragon Skin ${ }^{\mathrm{TM}}$ 20, Dragon Skin ${ }^{\mathrm{TM}}$ 30, Dragon Skin ${ }^{\mathrm{TM}}$ FX-Pro, Dragon Skin ${ }^{\mathrm{TM}}$ FX-Pro + Slacker, Ecoflex ${ }^{\mathrm{TM}}$ 0010, Ecoflex ${ }^{\mathrm{TM}}$ 00-30, Ecoflex ${ }^{\mathrm{TM}}$ 00-50, Rebound ${ }^{\mathrm{TM}}$ 25, Mold Star ${ }^{\mathrm{TM}} 16$ FAST, Mold Star ${ }^{\mathrm{TM}}$ 20T, SORTA-Clear ${ }^{\mathrm{TM}}$ 40, RTV615, PlatSil ${ }^{\circledR}$ Gel-10, Psycho Paint ${ }^{\circledR}$ and SOLOPLAST 150318 were subjected to uniaxial tensile tests according to the ASTM D412 standard. Sample preparation and tensile test parameters are described in detail. The tensile test data are used to derive parameters for hyperelastic material models using nonlinear least-squares methods, which are provided to the reader. This article presents the mechanical characterization and the resulting material properties for a wide set of commercially available hyperelastic materials, many of which are recognized and commonly applied in the field of soft robotics, together with some that have never been characterized. The experimental raw data and the algorithms employed to determine material parameters are shared on the Soft Robotics Materials Database GitHub repository to enable accessibility, as well as future contributions from the soft robotics community. The presented database is aimed at aiding soft roboticists in designing and modeling soft robots while providing a starting point for future material characterizations related to soft robotics research.
\end{abstract}

\section{Index Terms}

Body Double SILK, Dragon Skin 10 MEDIUM, Dragon Skin 20, Dragon Skin 30, Dragon Skin FX-Pro, Ecoflex 00-10, Ecoflex 00-30, Ecoflex 00-50, Rebound 25, Mold Star 16 FAST, Mold Star 20T, SORTA-Clear 40, RTV615, PlatSil Gel-10, Psycho Paint, SOLOPLAST 150318, Hyperelasticity, Constitutive model

\section{INTRODUCTION}

In recent years, soft robotics has become one of the fastest growing research fields in the robotic community. There has been an increasing interest in the use of unconventional compliant materials and non-standard actuators in robotic systems. A key challenge is the creation of structures that can vary their morphology and stiffness at will. But the efforts to invent new robots differ significantly from their conventional rigid counterparts. Despite an increase in the use of silicone rubbers in robotics design, the community lacks constitutive model parameters from the literature in comparison to the vast array of commercially available materials. Information commonly provided by manufacturers only contains Shore A hardness and tensile strength with few details on data acquisition and no access to raw data to determine model-specific parameters. Selecting a material based on such parameters alone is difficult, as simulation of soft robotics structures commonly requires further information on the material characteristics. To date, there is no collection of characteristic data for silicone rubbers relevant to soft robotics which could give indications of their relative behaviours. As pointed out by Paley [1], defining a catalog of target materials and properties would help the robotics community to address existing and future challenges in material supply.

Although useful for a specific structure, constitutive material models are often derived from a limited range of strain which are induced according to a particular application. At low strains (up to $50 \%$ extension/compression) the simple Hooke's law may be accurate enough to describe hyperelastic materials behaviour [2]. However, soft pneumatic actuators, often involve large strains [3]-[15], and therefore a more general hyperelastic model should be calibrated across a large range of strains appropriate for the application.

Characterization of elastomers with hyperelastic models has been reported in the literature within the context of soft robotics. The Smooth-On Ecoflex series, parts of the DragonSkin series and Sylgard184 are predominantly used in this framework. Atieh [16] characterized the Young's modulus for Ecoflex 00-10, Ecoflex 00-50, and Dragon Skin 20, but only for compression at low strain range. In [17], experimental stress-strain curves were obtained at a rate of $0.1 \mathrm{~mm} / \mathrm{min}$ for Ecoflex 00-50 and Dragon Skin 30 following a withdrawn standard. Case et al. [18] studied Sylgard184, Smooth-Sil 950 and Ecoflex 00-30 with specimens of thickness of $307 \mu \mathrm{m}, 643 \mu \mathrm{m}, 371 \mu \mathrm{m}$ respectively, and exhibited pull-to-failure data graph (at a rate of 100 $\mathrm{mm} / \mathrm{min}$ ), but did not provide parameters of constitutive models. Ogden model parameters in [19] were derived from tensile and compression tests on samples cured at $60{ }^{\circ} \mathrm{C}$ and pulled at $10 \mathrm{~mm} / \mathrm{min}$ speed deformation. Kulkarni [20] fitted Ecoflex 00-50

1Systems and Materials for Mechatronics (SYMME) Laboratory, University Savoie Mont Blanc, Annecy, France

2Department of Informatics, Haptics Mechatronics and Medical Robotics (HaMMeR) Laboratory, King's College London, London, UK

3UCL, EPSRC Centre for Interventional and Surgical Sciences (WEISS), London, UK

4Department of Surgery and Cancer, Imperial College London, London, UK 
with a Yeoh model, whereas Elsayed et al. [8], [21] used an Ogden model (with non-standard rectangular strips specimens at $300 \mathrm{~mm} / \mathrm{min}$ crosshead speed). Pineda [22] implemented a Mooney-Rivlin model for the latter material (on non-standard specimens with a bespoke automated setup at a speed of $0.2 \mathrm{~mm} / \mathrm{s}$ ) and so did Lee [23], but without providing information on the experimental conditions. Silicone Elastosil M4601 was characterized following a standard test method for tensile properties of plastics in [24], or on a $115 \mu \mathrm{m}$ thickness specimen in [25] with a Neo Hookean model. Only Low et al. [26] used a standardized protocol for Dragon Skin 10-Medium, as per the ASTM D412 [27] normative. This is a uniaxial tensile testing standard for obtaining material properties of elastomers.

In a pragmatic approach, FE analyses are often conducted in the field of soft robotics where mechanical responses of silicone rubbers are modeled as nonlinear elastic, isotropic and incompressible under quasi-static loading. In general, constitutive model parameters are typically derived from experiments conducted by uniaxially stretching the silicone rubber specimen in the principal loading direction. These assumptions and models can provide a sufficient basis to predict the behaviour of soft actuator designs and deliver reasonable agreement with the experimental results as in [5], [15], [21], [28]-[33]. However, in numerous studies, little information is provided on the experimental protocol, the measurement equipment, the specimens fabrication process or the nature of the data (true or engineering). It is therefore difficult to compare material models as no common reference methodology is currently used.

In this work, the following elastomers are tested and characterized: Body Double SILK, Dragon Skin 10 MEDIUM, Dragon Skin 20, Dragon Skin 30, Dragon Skin FX-Pro, Dragon Skin FX-Pro + Slacker, Ecoflex 00-10, Ecoflex 00-30, Ecoflex 00-50, Rebound 25, Mold Star 16 FAST, Mold Star 20T, SORTA-Clear 40, Psycho Paint (Smooth-On Inc., Easton PA.), RTV615 (Momentive Performance Materials Inc., Waterford NY), PlatSilGel-10 (Polytek Development Corp., Easton PA) and SOLOPLAST 150318 (Soloplast-Vosschemie, St Egrève FR.). Their characteristics from the manufacturer's datasheet are listed in Table I. Mechanical properties are derived from uniaxial tensile tests following the recommendations in ASTM D412 testing standards for vulcanized rubbers and elastomers. The choice of the best constitutive model and the values of the parameters are obtained through a minimization algorithm implemented in Python.

The aim of this work is to provide a common set of elastomeric material model parameters, as well as the corresponding raw data to benefit the soft robotics community and to help choosing appropriate materials for a considered application. We propose guidelines for experimental uniaxial tensile tests of elastomeric materials and provide detailed information on the mechanical properties of a large set of samples. The material parameters and subsequent hyperelastic models identified provide a basis for said materials to be used in the fabrication of soft robots. The methods in this work are presented in great detail to pave the way for a unified framework for material selection to the soft robotics community.

\section{Materials And Methods}

\section{A. Fabrication of the specimen}

The specimen's geometry and dimensions were prepared as indicated in method A of the ASTM D412 standard. Detailed information regarding the dumbbell specimen shape and testing specifications can be found in the aforementioned document. We used the "Die C" sample of thickness $3 \mathrm{~mm}$, with an overall length of $115 \mathrm{~mm}$ in which the narrow section is $33 \mathrm{~mm}$ long.A PRUSA i3 MK3S 3D printer (Prusa Research, Prague Cz) was used to generate the negative molds in which the liquid rubber was poured. The steps of the fabrication protocol are summarized in Figure 1. The preparation of the elastomer specimen is achieved by mixing equal weight of the two-part silicone rubber part A and part B (except for RTV615 (1A:10B) and SOLOPLAST 150318 (1A:50B) ) as recommended by the manufacturers. A Kern PCB 3500-2 (KERN \& SOHN GmbH, Balingen GE) electronic precision scale with an accuracy of $0.02 \mathrm{~g}$ is used for that purpose. The mixture was subjected to a vacuum for a period of $10 \mathrm{~min}$ to de-air (respectively $4 \mathrm{~min}$ for materials with very short pot life), then carefully poured into the molds with a syringe and cured at room temperature. The vacuum pump used was a Mastercool 90066-2V-220-A (Mastercool Inc., Randolph NJ) featuring Free Air Displacement of 170 1/min and Ultimate Vacuum of $75 \mu \mathrm{m}$. Each sample was visually inspected using a light source directed across the sample in order to identify possible remaining air bubbles within the material. Figure 2 shows a representative final sample that has been discarded due to the presence of one bubble spotted in the gauge-length. The thickness of the dumbbell specimens was measured using an OptoNCDT 1605 laser sensor (Micro-Epsilon, Ortenburg GE) to ensure the validity of the cross sectional area. Specimens not satisfying the aforementioned requirements were discarded from the tensile tests.

\section{B. Uniaxial tensile tests for incompressible hyperelastic materials}

1) Protocol: The tensile tests were carried out at $22{ }^{\circ} \mathrm{C}$ ambient temperature, with an Instron 5569 machine (Instron, Norwood Ma) equipped with a $1 \mathrm{kN}$ load cell of model 2525-806. The accuracy of the system is equal to or better than $0.025 \%$ of the cell rated output or $0.25 \%$ of the indicated load, depending on which is greater. At maximum, this yields an error of $0.25 \mathrm{~N}$ for readings under $100 \mathrm{~N}$. The global strain values were derived from the crosshead displacement readings. The displacement output recorded by the system is the sum of the system compliance and the specimen deformation. Crosshead, screw, and load cell deflections, grip or fixture deflections, are very small in comparison to the specimen deformation and thus 
were neglected for the measurement of the displacement [34]. A camera setup for measuring the local strain of the material is desirable. This, however, is not yet available in the current setup.

The placement of the samples inside the machine jaws requires special care. Guidelines to ensure the reliability of the tests are presented below:

- The surface area of the grip faces should be large enough to cover the tab of the dumbbell sample, as much of the surface area as possible. It should be ensured that the specimen end is gripped by at least $75 \%$ of the available jaw face length, otherwise gripping efficiency is reduced [34].

- The choice of the layout of the gripper mesh is important. A criss-cross fine serrated pattern gives better performances as it is specifically designed to improve friction. After preliminary trials with several gripping jaws, the mechanical wedge action grip of type 2710-010 from Instron, proved to have the best hold without any slippage. It can be seen in Figure 3

- Adding sandpaper sheets to the tab surface of the specimen should be avoided as it does not improve the grip and can in some cases lead to tear in the material.

- When positioning the sample inside the jaws, the sample protection should be activated on the machine to limit unwanted load on the specimen while manipulating. We set the limit load value to $0.1 \mathrm{~N}$. This security process is important as it prevents the sample from being stretched before the start of the test.

- It is advised to use a laser beam projected onto the surface of the specimen to ensure good alignment with the pulling direction without shear stresses. We used a QUIGO 3 device (Bosch, Gerlingen GE) for this purpose.

- Finally, the sample should be pre-loaded with $0.5 \mathrm{~N}$ (respectively $0.02 \mathrm{~N}$ for the softest materials: Ecoflex 00-10 and Dragon Skin Fx-Pro + Slacker) after being fixed in the grippers. These values were empirically obtained depending on the initial stiffness of the material. This was to ensure that the sample is not slack before starting with the extension. Omitting this crucial step would inevitably jeopardize the output of the strain-stretch curve.

2) Strain rate: When extending the sample, the grip separation shall be $500 \pm 50 \mathrm{~mm} / \mathrm{min}$. We used the maximum strain rate achievable with our machine of $450 \mathrm{~mm} / \mathrm{min}$ for the tensile tests. Additional tensile tests on separate samples at constant strain rates of 10,100, 200 and $300 \mathrm{~mm} / \mathrm{min}$ up to failure were also performed to evaluate possible dynamic effects on the stress-strain relationship, as well as investigate the possible effects that the norm is currently not considering with regards to soft robotics applications.

3) Tensile test repeatability: To determine reliability of the tensile test protocol, five different samples of the same material were pulled under the same conditions. The expression of the statistical dispersion can be calculated with a Type A evaluation of measurement uncertainty (i.e. standard deviation of the sample mean $s_{\bar{x}}$ ):

$$
s_{\bar{x}}=\frac{s}{\sqrt{n}}
$$

with $s$ being the corrected sample standard deviation:

$$
s=\sqrt{\frac{1}{n} \sum_{i=1}^{n}\left(y_{i}-\bar{y}_{i}\right)^{2}}
$$

where $n$ refers to the number of samples, $y_{i}$ the value of the measured true stress and $\bar{y}_{i}$ the arithmetic mean of the repeated true stress measured values.

The mean curve and a $95 \%$ confidence interval computed from the five tensile tests and the corrected sample standard deviation are used for fitting hyperelastic constitutive models from the data. Measurement was also found consistent and repeatable across the materials. As an example, Figure 4 illustrates the tensile test experimental data which are represented by the true stress-stretch curves for five specimens of Dragon Skin FX-Pro material.

\section{Principal Cauchy stress constitutive models}

Hyperelastic material models are often seen in the literature [35]-[37] relying upon the strain energy density function $W$ formulated in terms of strain invariants. However, in most commercial finite element analysis (FEA) software, relevant stressstrain data must be available as true stress and true strain data. We therefore derived the principal Cauchy stress as a function of stretch from the strain energy. This is a more relevant relationship for curve fitting purposes as tensile test machine data are in the form of stress and strain and do not directly provide the strain energy. Under the assumption of an isotropic incompressible behavior of the material under uniaxial loading, the principal Cauchy stress constitutive models as a function of invariants yields [36], [38]:

$$
\begin{aligned}
\sigma_{\text {true uniax }} & =2\left(\lambda^{2}-\frac{1}{\lambda}\right) \cdot\left(\frac{\partial W}{\partial I_{1}}+\frac{1}{\lambda} \frac{\partial W}{\partial I_{2}}\right) \\
\sigma_{\text {eng uniax }} & =2\left(\lambda-\frac{1}{\lambda^{-2}}\right) \cdot\left(\frac{\partial W}{\partial I_{1}}+\frac{1}{\lambda} \frac{\partial W}{\partial I_{2}}\right)
\end{aligned}
$$

where, the Cauchy-Green invariants $I_{1}$ and $I_{2}$, are in this particular case a function of the stretch $\lambda$ : 
$I_{1}=\lambda^{2}+\frac{2}{\lambda}, I_{2}=2 \lambda^{2}+\frac{1}{\lambda^{2}}$, with $\lambda=\exp \left(\varepsilon_{\text {true uniax }}\right)$

Constitutive hyperelastic models, including Neo-Hookean, Mooney-Rivlin, Yeoh, Ogden, Veronda-Westmann were used to determine material parameters in the incompressible isotropic hyperelastic stress-strain function on the basis of a nonlinear least squares optimization method using the Levenberg-Marquardt algorithm by fitting the data obtained from experiments. These models were selected as they are commonly available as constitutive models in FEA software, and perform well up to reasonably high strains for such materials. Table II summarizes each of these constitutive models.

1) Fitting Curve Algorithm: Fitting the curve means finding the constant parameters $\left(\mu, C_{i}\right.$, or $\left.\left(\mu_{i}, \alpha_{i}\right)\right)$ of the constitutive models listed in Table II that minimize an objective function $f$ that is a sum of squared errors between theoretical and experimental data. The error function is defined as:

$$
f=\sum_{i=1}^{N}\left[\sigma_{\text {predicted }, i}-\sigma_{\text {measured }, i}\right]^{2}
$$

where $N$ is the number of points. The optimization algorithm is implemented in Python using the least_squares function from the scipy.optimize package. This function has the ability to fit equations with multiple parameters to the data and utilizes the efficient Levenberg-Marquardt method to perform minimization. As an example, the code used to fit data with the Ogden model implemented Python is given in the Appendix. The full source code of our fitting algorithm is available on a GitHub repository [139].

2) Evaluation of the goodness of fit: The overall performance of each model numerical prediction is evaluated calculating correlation coefficients between experimental and theoretical data. As the coefficient of determination $R^{2}$ is only valid for linear regression, we instead used the Standard Error of the Estimate $S$ to quantify the goodness of fit. It represents the average distance that the observed values fall from the regression line by using the residual sum of squares and is defined as:

$$
S=\sqrt{\frac{\sum_{i=1}^{n}\left(y_{i}-\hat{y}_{i}\right)^{2}}{d f}}
$$

where $d f=n-k-1, \hat{y}_{i}$ refers to the predicted model value of true stress, $y_{i}$ the actual value and $d f$ the degree of freedom, and $n$ is the size of the sample number and $k$ the number of predictors (i.e. parameters to be estimated using the regression).

In addition, for the selection of the best model we used the Akaike Information Criterion $(A I C)$, a widely accepted measure for selecting nonlinear models within a cohort. It also accounts for the number of parameters in the model function and is computed as [40]:

$$
A I C=2 k+n \ln (L)
$$

with $k$ being the number of parameters in the model and $\ln (L)$ the maximum log-likelihood of the estimated model. The latter, is expressed in the case of nonlinear model fitting with least squares and normally distributed errors as:

$$
\ln (L)=\frac{1}{n} \sum_{i=1}^{n} \hat{\epsilon}_{i}
$$

$\hat{\epsilon}_{i}$ being the estimated residual sum of squares from the nonlinear least-squares fit and $n$ their number. The best model is then the one with the lowest $A I C$ which can take a negative value.

\section{RESULTS}

\section{A. Experimental data}

1) Stress-stretch curves: The experimental tensile stress-strain curves for the tested materials are shown in Figure 5. For the sake of clarity at low strain, the data are plotted as stress-stretch. Except for the Body Double SILK and PlatSilGel-10 and Psycho Paint and Rebound 25, each material has a distinct behavior from one another. The RTV615 and SORTA-Clear 40 are the stiffest materials, whereas the softest ones are the Ecoflex series. The Dragon Skin Series exhibits a relatively higher tensile stress at break (up to $50 \mathrm{MPa}$ for Dragon Skin FX-Pro) than the other materials as opposed to RTV615 and Body Double SILK material that break at relatively low tensile stress (less than $10 \mathrm{MPa}$ ).

2) Effect of strain rate: The stress-stretch curves up to rupture, for the different strain rates, for Psycho Paint and SOLOPLAST are shown in Figure 6. Within the range of low strains (up to approximately $150 \%$ strain) there is no significant difference in the material behavior. However, an effect of the tensile test rate in the region of large strain has been observed when the sample is pulled at low strain rate. As illustrated by Figure 6, the mechanical characteristics of some materials are more impacted by dynamic effects than others. For instance, only the $10 \mathrm{~mm} / \mathrm{min}$ strain rate produces a significant output for Psycho Paint as opposed to SOLOPLAST where two distinct behaviors of the material were observed between [10 - 100] and [200 - 450] $\mathrm{mm} / \mathrm{min}$ strain rate ranges.

${ }_{1}^{1}$ https://github.com/LucMarechal/Soft-Robotics-Materials-Database 


\section{B. Constitutive model parameters of tested elastomers}

Under the assumption of isotropic incompressible hyperelasticity behavior of the material, polynomial hyperelastic material models were investigated in the fitting of the tensile test data up to rupture. The accuracy of the constitutive models to predict the behavior of elastomers is illustrated, as an example in Figure 7, by comparison to the experimental data of the Ecoflex 00-10 material. The Mooney-Rivlin is in this case more accurate than the Yeoh, Neo-Hookean, and Veronda-Westmann models, but not as accurate as a 3-term Ogden model.

For all the tested materials, the numerical results are summarized in Table III] It provides optimal parameters for the different tested constitutive models. The most consistent model for each material is highlighted in bold. The Ogden model significantly outperformed the curve fitting for most of the cases. Exponential-based models (Veronda Westmann, Humphrey) did not allow for better predictions. The Neo-Hookean model failed to accurately represent the material behavior across all materials. The raw data obtained in this work is openly available [39] to be used as raw input for built-in native features of commercially available FE software or for further modelling approaches.

\section{DISCUSSION}

\section{A. Materials applicability}

This work has shown that hyperelastic materials cannot be selected only based on the manufacturers datasheets. For example, Rebound 25 and Body Double SILK present distinct responses to mechanical solicitation even though the Shore Hardness given by the manufacturer are identical. Besides, for some materials such as Psycho Paint or SOLOPLAST 150318 no details are provided for the mechanical properties. It is then necessary to have more accurate information such as the models derived from experimental tensile test in order to predict the material behavior and to compare them to each other. As for the sealing of two layers of materials in [41], in-house testing has shown that relatively stiff materials such as Rebound 25, Body Double SILK and Mold Star 16 Fast, have the advantage of bonding to Ecoflex and Dragon Skin series. Consequently, they can be used in conjunction to the latter to fix boundary conditions or locally rigidify the structure of a multilayer design, as in [12]. If the application requires large deformation, the decision as to which material to use needs careful consideration. The Ecoflex series has the ability to undergo large deformation under relatively small stress, which means it would require less pressure for a pneumatic or fluidic actuator to inflate a structure based on deformable membranes. Dragon Skin FX-Pro has among the highest tensile strength and elongation at break of the tested materials. This can have a significant practical implication in specific designs where a ballooning effect is expected. Moreover, when employed with Slacker at an adequate ratio, it becomes softer and sticky, which makes it an excellent candidate for realistic soft human tissue simulation.

In terms of appearance, especially for creating realistic tissue phantoms, it should be noted that the translucent materials can take any color by adding just a few drops of appropriate silicone color pigments. Conversely, SORTA-Clear 40 may be of interested in robotics design where water clear translucent optical properties are needed. The same applies to RTV615, which also has the advantage of featuring a low viscosity and long curing time. This might be useful in applications where the fabrication is tedious due to a time consuming pouring process or if mold cavities are difficult to reach with gravity alone.

\section{B. Constitutive models}

The constitutive model parameters provided in Table [III are valid for true stress - true strain data and therefore will give erroneous result if used for engineering data. Nonetheless, most of FEA software uses the true data. In this work, we fitted experimental data over the entire region of deformation with reasonable predictions. The parameters are given as an indication in order to have an overall view and a comparison of the behaviour of elastomers up to rupture. Attention is drawn to the fact that a higher accuracy is achievable if one fits the curves only for the appropriate range. Therefore, we recommend researchers to use the provided raw data, either engineering or true, and fit the curve according to their application. The python set of functions, available from the repository, allow for an easy implementation of the fitting algorithm.

Multiple factors could potentially affect the mechanical properties of hyperelastic materials, such as the curing temperature [42], ultraviolet degradation, natural aging, mixing ratio or the use of silicone pigment. These factors were not at stake in this study as it only considered materials cured at room temperature with the nominal mixing ratio recommended by the manufacturers. In this work we focused on characterising the nonlinear elastic response of materials and did not explore viscoelastic behaviours or damage incurred during previous loading. Moreover, the damage incurred during previous loading of the elastomer component has not been investigated with, for example, the analysis of the Mullins effect. However, this intrinsic property, resulting in softening, should be an important consideration in material choice of soft robots. The stress-stretch curves can see large changes between the initial pull and subsequent pulls, which is going to affect the force output possible by soft actuators and load bearing of highly deformable soft structures. The latter curves after the initial pull are arguably more important for soft robotics than the initial curve when considering systems that are going to experience cyclic behavior. It would then be advantageous to implement Ogden-Roxburgh [43] or Qi-Boyce [44] models, which extend hyperelastic material models and account for the Mullins effect.

\footnotetext{
${ }^{2}$ https://github.com/LucMarechal/Soft-Robotics-Materials-Database
} 


\section{Limitations}

As with most tensile testing-based material characterisation conducted for soft robotics applications, this work considered the global strain induced in the investigated rubber materials. This, however, often presents itself as a limitation, as local strain observable in the gauge region of the sample may be larger than the global strain. Hence, the measurement could potentially result in an underestimate of the induced strain and consequently an overestimate of the stiffness of the material. Obtaining an accurate and reliable independent strain measurement for these highly compliant elastomers is still the subject of ongoing research. The use of mechanical extensometers can induce undesirable stresses. Non-contact techniques with digital image correlation (DIC) are preferable but can be tedious to implement meticulously and are still prone to errors [45], especially for large deformations. In the near future, a way to improve strain measurements for very large deformations will be investigated using a stereo high-speed camera and Kisdef-7D [46], an image correlation software that has shown promising capabilities with an elastomer [47].

Characterizing and comparing hyperelastic materials is particularly challenging due to the effect of hysteresis, the Mullins effect, stress-softening or strain rate sensitivity, which can be difficult to separate from each other. In the field of Soft Robotics, despite growing interest, few applications employing dynamic FEM models or taking into account the consequences of viscoelastic effects in the design are reported in the literature [48], [49]. In numerous soft robotics applications, actuators are often designed to reach a specific strain. When the stress remains constant or where the control involves a critical repeated pattern of material loading and unloading, this can be potentially problematic if the effects of viscoelastic behaviour are significant. To account for such behaviour, mechanical testing should involve creep-recovery tests (loading the material at constant stress, holding that stress for some time and then removing the load), stress-relaxation tests (straining the material at constant strain and then holding that strain) or cyclic test (repeating a pattern of loading-unloading).

Tensile tests were only performed under monotonic strain rate of $450 \mathrm{~mm} / \mathrm{min}$ up to rupture as per the employed standard. However, it was observed that the strain rate may have an effect on the stress strain behaviour of some of the materials, particularly for high strains, whereas the constitutive models presented in this work do not account for it. This is currently one of the limitations of this standard, an extension of which with an additional strain rates could benefit in-line applications with soft robotics. Hence, if simulations are required for applications which involve these effects, the generally-applicable results presented in this work should be used as a basis for additional, application-specific material testing.

It must be acknowledged that uniaxial loading tensile test alone may not rigorously represent the real behaviour for all load cases. However, an exhaustive characterization would require a considerable amount of experimental data and significant equipment to determine a set of strain-stress curves for a whole spectrum of loading cases. For many practical applications, the downside of adding model complexity is that it requires more experimental data for proper calibration, it is very computationally expensive and slow to simulate as pointed out in [38], [50], especially when soft robot topologies are intricate and fine meshes are involved. Shear tests, biaxial tests, equibiaxial or torsion tests are other kinds of stresses which could be complementary to characterize the material and provide an enhanced calibration of a hyperelastic model.

\section{CONClusion}

This article aims to offer an insight into the mechanical characteristics of a wide range of elastomers for soft robotics applications. Relying on the ASTM D412 testing standards, we followed a rigorous protocol to characterize elastomers through tensile testing. It was shown that hyperelastic models can be derived from experimental tensile test data. Some predictive models fit better than others. This is in particular the case for the Ogden model that proved to be the most accurate overall as opposed to the Neo Hookean model that showed the worst performance. Seventeen types of silicone materials namely Body Double SILK, Dragon Skin 10 MEDIUM, Dragon Skin 20, Dragon Skin 30, Dragon Skin FX-Pro, Dragon Skin FX-Pro + Slacker, Ecoflex 0010, Ecoflex 00-30, Ecoflex 00-50, Rebound 25, Mold Star 16 FAST, Mold Star 20T, SORTA-Clear 40, RTV615, PlatSilGel-10, Psycho Paint and SOLOPLAST 150318 have been investigated and constitutive models and their coefficients have also been derived. This work will help researchers to choose appropriate materials for their applications, determine a respective modelling approach, run comparative Finite Element simulations and pave the way to new designs in a more predictive way.

For the sake of generality and reproducibility, there is a need to unify methods for fabrication, experimental testing and characterization of elastomers within the framework of soft robotics applications. Researchers in the community should follow international standards to get a common reference of material characteristics and models. Therefore, we have started to create an accessible resource around soft robotics with the presented work. The mechanical properties and constitutive model parameters of various elastomers are listed in an open source database. The tensile tests data and the curve fitting algorithm are available to the community from its GitHub repository. We will advance this work with supplementary materials and encourage the community to contribute to the consolidation of this collaborative database by applying the proposed set of methods.

An extension of this initial study for the near future is the characterization of the mechanical response of the selected silicone rubbers with an independent measurement of the strain and considering other loading scenarios. We therefore plan to perform biaxial tensile tests and cyclic tension tests with increased values of deformation. Observations of viscosity-induced rate-dependent phenomena also provide motivation for considering relaxation experiments. Assessments of other generalized constitutive models will be pursued to take into account additional material properties. 


\section{ACKNOWLEDGMENTS}

We would like to gratefully acknowledge support of this work by Cancer Research UK (CRUK) [Award Reference: C63674/A26161]

\section{Author Disclosure StATEMENT}

No competing financial interests exist.

\section{REFERENCES}

[1] Paley DA, Majidi C, Tytell E, Wereley N. Bioinspired soft robotics: preface to the special issue. Bioinspiration \& Biomimetics. 2016;11(2).

[2] Shiva A, Sadati SMH, Noh Y, Fraś J, Ataka A, Würdemann H, et al. Elasticity Versus Hyperelasticity Considerations in Quasistatic Modeling of a Soft Finger-Like Robotic Appendage for Real-Time Position and Force Estimation. Soft Robotics. 2019;6(2).

[3] Patel DK, Sakhaei AH, Layani M, Zhang B, Ge Q, Magdassi S. Highly Stretchable and UV Curable Elastomers for Digital Light Processing Based 3D Printing. Advanced Materials. 2017;29(15).

[4] Cheng S, Narang YS, Yang C, Suo Z, Howe RD. Stick-On Large-Strain Sensors for Soft Robots. Advanced Materials Interfaces. 2019;6(20):1900985.

[5] Martinez RV, Branch JL, Fish CR, Jin L, Shepherd RF, Nunes RMD, et al. Robotic Tentacles with Three-Dimensional Mobility Based on Flexible Elastomers. Advanced Materials. 2013;25(2):205-212.

[6] Digumarti KM, Conn AT, Rossiter J. EuMoBot: replicating euglenoid movement in a soft robot. Journal of The Royal Society Interface. 2018 nov;15(148).

[7] Shepherd RF, Ilievski F, Choi W, Morin SA, Stokes AA, Mazzeo AD, et al. Multigait soft robot. Proceedings of the National Academy of Sciences. 2011;108(51):20400-20403.

[8] Elsayed Y, Vincensi A, Lekakou C, Geng T, Saaj CM, Ranzani T, et al. Finite Element Analysis and Design Optimization of a Pneumatically Actuating Silicone Module for Robotic Surgery Applications. Soft Robotics. 2014;1(4):255-262.

[9] Agarwal G, Besuchet N, Audergon B, Paik J, Walsh CJ. Stretchable Materials for Robust Soft Actuators towards Assistive Wearable Devices. Scientific Reports. 2016;6(1):34224.

[10] Miriyev A, Stack K, Lipson H. Soft material for soft actuators. Nature Communications. 2017;8(1):596.

[11] Kim S, Laschi C, Trimmer B. Soft robotics: a bioinspired evolution in robotics. Trends in Biotechnology. 2013;31(5):287-294.

[12] Marchese AD, Katzschmann RK, Rus D. A Recipe for Soft Fluidic Elastomer Robots. Soft Robotics. 2015;2(1):7-25.

[13] Rus D, Tolley MT. Design, fabrication and control of soft robots. Nature. 2015;521(7553):467-475.

[14] Joshi A, Kulkarni A, Tadesse Y. FludoJelly: Experimental study on jellyfish-like soft robot enabled by soft pneumatic composite (SPC). Robotics. 2019;8(3):56.

[15] Yan J, Dong H, Zhang X, Zhao J. A three-chambed soft actuator module with omnidirectional bending motion. In: 2016 IEEE International Conference on Real-time Computing and Robotics (RCAR); 2016. p. 505-510.

[16] Atieh A. Design, Modeling, Fabrication and Testing of a Piezoresistive-Based Tactile Sensor for Minimally Invasive Surgery Applications. Concordia University, Montreal, Quebec, Canada; 2012.

[17] Ranzani T, Gerboni G, Cianchetti M, Menciassi A. A bioinspired soft manipulator for minimally invasive surgery. Bioinspiration \& Biomimetics. 2015;10(3):35008

[18] Case JC, White EL, Kramer RK. Soft Material Characterization for Robotic Applications. Soft Robotics. 2015;2(2):80-87.

[19] Paternò L, Tortora G, Menciassi A. Hybrid Soft-Rigid Actuators for Minimally Invasive Surgery. Soft Robotics. 2018;5(6):783-799.

[20] Kulkarni P. Centrifugal Forming and Mechanical Properties of Silicone-based Elastomers for Soft Robotic Actuators. Graduate School-New Brunswick; 2015.

[21] Elsayed Y, Lekakou C, Geng T, Saaj CM. Design optimisation of soft silicone pneumatic actuators using finite element analysis. In: 2014 IEEE/ASME International Conference on Advanced Intelligent Mechatronics. IEEE; 2014. p. 44-49.

[22] Pineda F, Bottausci F, Icard B, Malaquin L, Fouillet Y. Using electrofluidic devices as hyper-elastic strain sensors: Experimental and theoretical analysis Microelectronic Engineering. 2015;144:27-31.

[23] Lee JK, Stoffel N, Fite K. Electronic packaging of sensors for lower limb prosthetics. In: 2012 IEEE 62nd Electronic Components and Technology Conference; 2012. p. 86-91.

[24] Wang T, Ge L, Gu G. Programmable design of soft pneu-net actuators with oblique chambers can generate coupled bending and twisting motions. Sensors and Actuators A: Physical. 2018;271:131-138.

[25] Alici G, Canty T, Mutlu R, Hu W, Sencadas V. Modeling and Experimental Evaluation of Bending Behavior of Soft Pneumatic Actuators Made of Discrete Actuation Chambers. Soft Robotics. 2018;5(1):24-35.

[26] Low JH, Ang MH, Yeow CH. Customizable soft pneumatic finger actuators for hand orthotic and prosthetic applications. In: 2015 IEEE International Conference on Rehabilitation Robotics (ICORR). IEEE; 2015. p. 380-385.

[27] ASTM-D412-16. Standard Test Methods for Vulcanized Rubber and Thermoplastic Elastomers-Tension. West Conshohocken, PA, www.astm.org: ASTM International; 2016

[28] Zhang J, Tang J, Hong J, Lu T, Wang H. The Design and Analysis of Pneumatic Rubber Actuator of Soft Robotic Fish. In: Zhang X, Liu H, Chen Z, Wang N, editors. Intelligent Robotics and Applications. Cham: Springer International Publishing; 2014. p. 320-327.

[29] Polygerinos P, Wang Z, Overvelde JTB, Galloway KC, Wood RJ, Bertoldi K, et al. Modeling of Soft Fiber-Reinforced Bending Actuators. IEEE Transactions on Robotics. 2015;31(3):778-789.

[30] Suzumori K, Endo S, Kanda T, Kato N, Suzuki H. A Bending Pneumatic Rubber Actuator Realizing Soft-bodied Manta Swimming Robot. In: Proceedings 2007 IEEE International Conference on Robotics and Automation; 2007. p. 4975-4980.

[31] Wakimoto S, Suzumori K, Ogura K. Miniature Pneumatic Curling Rubber Actuator Generating Bidirectional Motion with One Air-Supply Tube. Advanced Robotics. 2011;25(9-10):1311-1330.

[32] du Pasquier C, Chen T, Tibbits S, Shea K. Design and Computational Modeling of a 3D Printed Pneumatic Toolkit for Soft Robotics. Soft Robotics. 2019;6(5):657-663.

[33] Huri D, Mankovits T. Comparison of the material models in rubber finite element analysis. IOP Conference Series: Materials Science and Engineering. 2018;393:012018.

[34] INSTRON. 7 Tips for Materials Testing; 2007. [Accessed 3rd March 2019]. [Online]. Available from: = https://instron.com/-/media/literaturelibrary/whitepapers/2007/03/7-tips-for-materials-testing.pdf.

[35] Martins PALS, Natal Jorge RM, Ferreira AJM. A Comparative Study of Several Material Models for Prediction of Hyperelastic Properties: Application to Silicone-Rubber and Soft Tissues. Strain. 2006;42(3):135-147.

[36] Rackl M. Material testing and hyperelastic material model curve fitting for Ogden, Polynomial and Yeoh models. In: ScilabTEC (7 th International Scilab Users Conference). Paris; 2015. .

[37] Ogden RW, Saccomandi G, Sgura I. Fitting hyperelastic models to experimental data. Computational Mechanics. 2004 nov;34(6):484-502. 
[38] Bergström J. Elasticity/Hyperelasticity. In: Mechanics of Solid Polymers: Theory and Computational Modeling. William Andrew Publishing; 2015. p. 209-307.

[39] Marechal L. Soft Robotics Materials. Zenodo; 2020. Available from: https://doi.org/10.5281/zenodo.3611329

[40] Burnham KP, Anderson DR. Model Selection and Multimodel Inference: A Practical Information-Theoretic Approach. 2nd ed. Springer-Verlag New York; 2002

[41] Lindenroth L, Housden RJ, Wang S, Back J, Rhode K, Liu H. Design and integration of a parallel, soft robotic end-effector for extracorporeal ultrasound. IEEE Transactions on Biomedical Engineering. 2019;p. 1.

[42] Johnston ID, McCluskey DK, Tan CKL, Tracey MC. Mechanical characterization of bulk Sylgard 184 for microfluidics and microengineering. Journal of Micromechanics and Microengineering. 2014;24(3):035017.

[43] Ogden RW, Roxburgh DG. A Pseudo-Elastic Model for the Mullins Effect in Filled Rubber. Proceedings: Mathematical, Physical and Engineering Sciences. 1999;455(1988):2861-2877.

[44] Qi HJ, Boyce MC. Constitutive model for stretch-induced softening of the stress?stretch behavior of elastomeric materials. Journal of Mechanics Physics of Solids. 2004;52(10):2187-2205.

[45] Zhao J, Sang Y, Duan F. The state of the art of two-dimensional digital image correlation computational method. Engineering Reports. 2019;1(2):e12038.

[46] Vacher P, Dumoulin S, Morestin F, Mguil-Touchal S. Bidimensional strain measurement using digital images. Proceedings of the Institution of Mechanical Engineers, Part C: Journal of Mechanical Engineering. 1999;213(8):811-817.

[47] Meunier L, Chagnon G, Favier D, Orgéas L, Vacher P. Mechanical experimental characterisation and numerical modelling of an unfilled silicone rubber. Polymer Testing. 2008;27(6):765-777.

[48] Matia Y, Gat AD. Dynamics of Elastic Beams with Embedded Fluid-Filled Parallel-Channel Networks. Soft robotics. 2015;2(1):42-47.

[49] Moseley P, Florez JM, Sonar HA, Agarwal G, Curtin W, Paik J. Modeling, Design, and Development of Soft Pneumatic Actuators with Finite Element Method. Advanced Engineering Materials. 2016;18(6):978-988.

[50] Lipson H. Challenges and Opportunities for Design, Simulation, and Fabrication of Soft Robots. Soft Robotics. 2014;1(1):21-27. 
TABLE I

LIST AND INFORMATION ON TESTED MATERIALS

\begin{tabular}{lclccl}
\hline Material & Shore Hardness & Color & Pot life (min) & Cure time (min) & Manufacturer \\
\hline SORTA-Clear 40 & 40A & Water Clear Translucent & 60 & 960 & Smooth-On \\
Dragon Skin 10 MEDIUM & 10A & Translucent & 20 & 300 & Smooth-On \\
Dragon Skin 20 & $20 \mathrm{~A}$ & Translucent & 25 & 240 & Smooth-On \\
Dragon Skin 30 & $30 \mathrm{~A}$ & Translucent & 45 & 960 & Smooth-On \\
Dragon Skin Fx-Pro & $2 \mathrm{~A}$ & Translucent & 12 & 40 & Smooth-On \\
Dragon Skin Fx-Pro + Slacker & N/K & Yellow & 12 & 40 & Smooth-On \\
Ecoflex 00-10 & $00-10$ & Translucent & 30 & 240 & Smooth-On \\
Ecoflex 00-30 & $00-30$ & Translucent & 45 & 240 & Smooth-On \\
Ecoflex 00-50 & 00-50 & Translucent & 18 & 180 & Smooth-On \\
PlatSilGel-10 & $10 \mathrm{~A}$ & Milky White & 6 & 30 & Polytek \\
RTV615 & $44 \mathrm{~A}$ & Water Clear Translucent & 240 & 8640 & Momentive \\
Rebound 25 & $25 \mathrm{~A}$ & Orange & 20 & 320 & Smooth-On \\
Body Double SILK & $25 \mathrm{~A}$ & Green & 6 & 20 & Smooth-On \\
Mold Star 16 FAST & $16 \mathrm{~A}$ & Blue-Green & 6 & 30 & Smooth-On \\
Mold Star 20T & $20 \mathrm{~A}$ & Translucent & 6 & 30 & Smooth-On \\
Psycho Paint & N/K & Translucent Clear & 45 & N/K & Smooth-On \\
SOLOPLAST 150318 & N/K & White & $5-180$ & N/K & Soloplast-Vosschemie \\
\hline
\end{tabular}

TABLE II

INCOMPRESSIBLE HYPERELASTIC STRAIN ENERGY FUNCTIONS AND PRINCIPAL CAUCHY STRESS CONSTITUTIVE MODEL

\begin{tabular}{lll}
\hline Model & Strain energy density function & Principal Cauchy stresses \\
\hline Neo-Hookean & $W=\mu\left(I_{1}-3\right)$ & $\sigma_{\text {uniax }}=\mu\left(\lambda^{2}-\frac{1}{\lambda}\right)$ \\
Mooney-Rivlin & $W=C_{10}\left(I_{1}-3\right)+C_{01}\left(I_{2}-3\right)+C_{20}\left(I_{1}-3\right)^{2}$ & $\sigma_{\text {uniax }}=2\left(\lambda^{2}-\frac{1}{\lambda}\right)\left[C_{10}+\frac{C_{01}}{\lambda}+2 C_{20}\left(\lambda^{2}+\frac{2}{\lambda}-3\right)\right]$ \\
Yeoh & $W=\sum_{i=1}^{n} 2\left(\lambda-\lambda^{-2}\right) \cdot i \cdot C_{i}\left(I_{1}-3\right)^{i-1}$ & $\sigma_{\text {uniax }}=2\left(\lambda^{2}-\frac{1}{\lambda}\right) \sum_{i=1}^{n} i \cdot C_{i}\left(I_{1}-3\right)^{i-1}$ \\
Ogden & $W=\sum_{p=1}^{n} \frac{\mu_{p}}{\alpha_{p}}\left[\lambda_{1}^{\alpha_{p}}+\lambda_{2}^{\alpha_{p}}+\lambda_{3}^{\alpha_{p}}-3\right]$ & $\sigma_{\text {uniax }}=\sum_{p=1}^{n} 2 \mu_{p}\left[\lambda^{\left(\alpha_{p}-1\right)}-\lambda^{-\left(\frac{1}{2} \alpha_{p}+1\right)}\right]$ \\
Humphrey & $W=C_{1}\left(e^{C_{2}\left(I_{1}-3\right)}-1\right)$ & $\sigma_{\text {uniax }}=2\left(\lambda^{2}-\frac{1}{\lambda}\right) C_{1} C_{2}\left[e^{C_{2}\left(I_{1}-3\right)}\right]$ \\
Veronda-Westmann & $W=C_{1}\left(e^{C_{2}\left(I_{1}-3\right)}\right)-\frac{C_{1} C_{2}}{2}\left(I_{2}-3\right)$ & $\sigma_{\text {uniax }}=2\left(\lambda^{2}-\frac{1}{\lambda}\right) C_{1} C_{2}\left[e^{C_{2}\left(I_{1}-3\right)}-\frac{1}{2 \lambda}\right]$ \\
\hline
\end{tabular}

n: model order 
TABLE III

CONSTITUTIVE MODEL PARAMETERS (THE UNITS OF PARAMETERS ARE EXPRESSED IN MPA)

\begin{tabular}{|c|c|c|c|c|c|c|}
\hline Material & Neo-Hookean & Mooney-Rivlin & Yeoh & Ogden & Veronda-Westmann & Humphrey \\
\hline $\begin{array}{l}\text { Ecoflex } 00-10 \\
(\mathrm{~S}, \mathrm{AIC})\end{array}$ & $\begin{array}{l}\mu=0.02264 \\
(0.89,-175.0)\end{array}$ & $\begin{array}{l}C_{10}=2.18 \times 10^{-2} \\
C_{01}=-7.22 \times 10^{-2} \\
C_{20}=1.26 \times 10^{-5} \\
(0.08,-3794.4)\end{array}$ & $\begin{array}{l}C_{1}=1.00 \times 10^{-3} \\
C_{2}=1.31 \times 10^{-2} \\
C_{3}=2.056 \times 10^{-5} \\
(0.20,-2376.1)\end{array}$ & $\begin{array}{l}\mu_{1}=0.7363, \alpha_{1}=2.858 \\
\mu_{2}=0.8074, \alpha_{2}=2.604 \\
\mu_{3}=-1.526, \alpha_{3}=2.740 \\
(\mathbf{0 . 0 2},-\mathbf{5 8 4 2 . 3})\end{array}$ & $\begin{array}{l}C_{1}=9.028 \\
C_{2}=1.73 \times 10^{-3} \\
(0.2,-2132.9)\end{array}$ & $\begin{array}{l}C_{1}=8.048 \\
C_{2}=1.829 \times 10^{-3} \\
(0.26,-2010.9)\end{array}$ \\
\hline $\begin{array}{l}\text { Ecoflex 00-30 } \\
(\mathrm{S}, \mathrm{AIC})\end{array}$ & $\begin{array}{l}\mu=0.03245 \\
(1.66,719.7)\end{array}$ & $\begin{array}{l}C_{10}=1.80 \times 10^{-2} \\
C_{01}=-4.69 \times 10^{-2} \\
C_{20}=4.34 \times 10^{-5} \\
(0.09,-3437.5)\end{array}$ & $\begin{array}{l}C_{1}=1.00 \times 10^{-1} \\
C_{2}=1.20 \times 10^{-2} \\
C_{3}=4.96 \times 10^{-5} \\
(0.14,-2723.1)\end{array}$ & $\begin{array}{l}\mu_{1}=-0.322, \alpha_{1}=3.310 \\
\mu_{2}=0.19, \alpha_{2}=3.115 \\
\mu_{3}=0.145, \alpha_{3}=3.468 \\
(0.02,-5391.1)\end{array}$ & $\begin{array}{l}C_{1}=5.79 \\
C_{2}=3.05 \times 10^{-3} \\
(0.29,-1714.7)\end{array}$ & $\begin{array}{l}C_{1}=5.26 \\
C_{2}=3.16 \times 10^{-3} \\
(0.31,-1613.5)\end{array}$ \\
\hline $\begin{array}{l}\text { Ecoflex } 00-50 \\
(\mathrm{~S}, \mathrm{AIC})\end{array}$ & $\begin{array}{l}\mu=0.048176 \\
(2.21,1148.4)\end{array}$ & $\begin{array}{l}C_{10}=3.53 \times 10^{-2} \\
C_{01}=-1.05 \times 10^{-1} \\
C_{20}=4.77 \times 10^{-5} \\
(0.17,-2517.3)\end{array}$ & $\begin{array}{l}C_{1}=1.00 \times 10^{-1} \\
C_{2}=2.22 \times 10^{-2} \\
C_{3}=6.07 \times 10^{-5} \\
(0.32,-1653.5)\end{array}$ & $\begin{array}{l}\mu_{1}=1.970, \alpha_{1}=2.911 \\
\mu_{2}=-3.671, \alpha_{2}=3.008 \\
\mu_{3}=1.740, \alpha_{3}=3.096 \\
(0.05,-4226.9)\end{array}$ & $\begin{array}{l}C_{1}=1.18 \times 10^{1} \\
C_{2}=2.46 \times 10^{-3} \\
(0.47,-1071.2)\end{array}$ & $\begin{array}{l}C_{1}=1.06 \times 10^{1} \\
C_{2}=2.57 \times 10^{-3} \\
(0.51,-965.2)\end{array}$ \\
\hline $\begin{array}{l}\text { PlatSilGel-10 } \\
\text { (S, AIC) }\end{array}$ & $\begin{array}{l}\mu=0.090681 \\
(0.61,-460.7)\end{array}$ & $\begin{array}{l}C_{10}=1.02 \times 10^{-1} \\
C_{01}=-1.41 \times 10^{-1} \\
C_{20}=1.93 \times 10^{-5} \\
(0.03,-3148.6)\end{array}$ & $\begin{array}{l}C_{1}=1.00 \times 10^{-1} \\
C_{2}=7.60 \times 10^{-2} \\
C_{3}=7.81 \times 10^{-5} \\
(0.23,-1351.1)\end{array}$ & $\begin{array}{l}\mu_{1}=-6.820 \times 10^{-1}, \alpha_{1}=2.613 \\
\mu_{2}=4.088 \times 10^{-7}, \alpha_{2}=6.539 \\
\mu_{3}=6.547 \times 10^{-1}, \alpha_{3}=2.752 \\
(0.02,-3538.5)\end{array}$ & $\begin{array}{l}C_{1}=6.414 \times 10^{1} \\
C_{2}=1.31 \times 10^{-3} \\
(0.18,-1584.2)\end{array}$ & $\begin{array}{l}C_{1}=4.48 \times 10^{1} \\
C_{2}=1.72 \times 10^{-3} \\
(0.24,-1304.6)\end{array}$ \\
\hline $\begin{array}{l}\text { SORTA-Clear } 40 \\
(\mathrm{~S}, \mathrm{AIC})\end{array}$ & $\begin{array}{l}\mu=0.290228 \\
(0.98,-9.5)\end{array}$ & $\begin{array}{l}C_{10}=3.53 \times 10^{-1} \\
C_{01}=-4.23 \times 10^{-1} \\
C_{20}=4.79 \times 10^{-4} \\
(\mathbf{0 . 1 5}, \mathbf{- 8 5 1 . 5 )}\end{array}$ & $\begin{array}{l}C_{1}=1.00 \times 10^{-1} \\
C_{2}=2.11 \times 10^{-1} \\
C_{3}=1.66 \times 10^{-3} \\
(0.34,-468.3)\end{array}$ & $\begin{array}{l}\mu_{1}=-4.360 \times 10^{-2}, \alpha_{1}=3.561 \\
\mu_{2}=3.549 \times 10^{-2}, \alpha_{2}=3.561 \\
\mu_{3}=3.689 \times 10^{-2}, \alpha_{3}=3.561 \\
(0.21,-685.3)\end{array}$ & $\begin{array}{l}C_{1}=29.83 \\
C_{2}=8.712 \times 10^{-3} \\
(0.30,-523.2)\end{array}$ & $\begin{array}{l}C_{1}=19.42 \\
C_{2}=1.134 \times 10^{-2} \\
(0.38,-419.3)\end{array}$ \\
\hline RTV 615 & $\begin{array}{l}\mu=0.33861 \\
(0.66,-69.4)\end{array}$ & $\begin{array}{l}C_{10}=-2.02 \times 10^{-2} \\
C_{01}=2.51 \times 10^{-1} \\
C_{20}=3.01 \times 10^{-2} \\
(0.02,-642.9)\end{array}$ & $\begin{array}{l}C_{1}=1.00 \times 10^{-1} \\
C_{2}=1.13 \times 10^{-1} \\
C_{3}=2.45 \times 10^{-2} \\
(0.06,-475.3)\end{array}$ & $\begin{array}{l}\mu_{1}=1.292, \alpha_{1}=4.999 \\
\mu_{2}=1.637, \alpha_{2}=4.577 \\
\mu_{3}=-2.828, \alpha_{3}=4.799 \\
(0.009,-776.4)\end{array}$ & $\begin{array}{l}C_{1}=1.656 \\
C_{2}=1.36 \times 10^{-1} \\
(0.02,-653.1)\end{array}$ & $\begin{array}{l}C_{1}=1.095 \\
C_{2}=1.55 \times 10^{-1} \\
(0.03,-601.2)\end{array}$ \\
\hline $\begin{array}{l}\text { Body Double SILK } \\
(\mathrm{S}, \mathrm{AIC})\end{array}$ & $\begin{array}{l}\mu=0.07868 \\
(0.13,-1039.2)\end{array}$ & $\begin{array}{l}C_{10}=7.37 \times 10^{-2} \\
C_{01}=-1.38 \times 10^{-2} \\
C_{20}=1.18 \times 10^{-4} \\
(0.01,-2260.9)\end{array}$ & $\begin{array}{l}C_{1}=1.00 \times 10^{-1} \\
C_{2}=6.95 \times 10^{-2} \\
C_{3}=1.45 \times 10^{-4} \\
(0.02,-2089.5)\end{array}$ & $\begin{array}{l}\mu_{1}=-3.099 \times 10^{-1}, \alpha_{1}=18.341 \\
\mu_{2}=2.557 \times 10^{-1}, \alpha_{2}=2.729 \\
\mu_{3}=1.036 \times 10^{-1}, \alpha_{3}=2.649 \\
(0.01,-2268.6)\end{array}$ & $\begin{array}{l}C_{1}=43.54 \\
C_{2}=1.86 \times 10^{-3} \\
(0.02,-2004.2)\end{array}$ & $\begin{array}{l}C_{1}=18.84 \\
C_{2}=3.71 \times 10^{-3} \\
(0.02,-2034.2)\end{array}$ \\
\hline $\begin{array}{l}\text { Rebound } 25 \\
\text { (S, AIC) }\end{array}$ & $\begin{array}{l}\mu=0.154681 \\
(0.91,-53.1)\end{array}$ & $\begin{array}{l}C_{10}=1.66 \times 10^{-1} \\
C_{01}=-2.28 \times 10^{-1} \\
C_{20}=3.80 \times 10^{-4} \\
(0.08,-1380.9)\end{array}$ & $\begin{array}{l}C_{1}=1.00 \times 10^{-1} \\
C_{2}=1.00 \times 10^{-1} \\
C_{3}=7.46 \times 10^{-4} \\
(0.23,-807.4)\end{array}$ & $\begin{array}{l}\mu_{1}=7.714 \times 10^{-1}, \alpha_{1}=3.059 \\
\mu_{2}=-1.5223, \alpha_{2}=3.003 \\
\mu_{3}=7.441 \times 10^{-1}, \alpha_{3}=3.059 \\
(\mathbf{0 . 0 4},-1797.7)\end{array}$ & $\begin{array}{l}C_{1}=15.21 \\
C_{2}=8.188 \times 10^{-3} \\
(0.23,-818.4)\end{array}$ & $\begin{array}{l}C_{1}=11.22 \\
C_{2}=9.671 \times 10^{-3} \\
(0.28,-700.7)\end{array}$ \\
\hline $\begin{array}{l}\text { DragonSkin FX-Pro } \\
(\mathrm{S}, \mathrm{AIC})\end{array}$ & $\begin{array}{l}\mu=0.074198 \\
(2.68,1432.9)\end{array}$ & $\begin{array}{l}C_{10}=6.82 \times 10^{-2} \\
C_{01}=-1.99 \times 10^{-1} \\
C_{20}=4.66 \times 10^{-5} \\
(0.24,-2071.0)\end{array}$ & $\begin{array}{l}C_{1}=1.00 \times 10^{-1} \\
C_{2}=4.35 \times 10^{-2} \\
C_{3}=7.07 \times 10^{-5} \\
(0.56,-824.7)\end{array}$ & $\begin{array}{l}\mu_{1}=-0.1658, \alpha_{1}=3.275 \\
\mu_{2}=0.4020, \alpha_{2}=3.309 \\
\mu_{3}=-0.2390, \alpha_{3}=3.275 \\
(0.85,-3580.3)\end{array}$ & $\begin{array}{l}C_{1}=28.44 \\
C_{2}=1.81 \times 10^{-3} \\
(0.67,-571.3)\end{array}$ & $\begin{array}{l}C_{1}=25.24 \\
C_{2}=1.92 \times 10^{-3} \\
(0.74,-439.5)\end{array}$ \\
\hline $\begin{array}{l}\text { Dragon Skin } 10 \text { MEDIUM } \\
(\mathrm{S}, \mathrm{AIC})\end{array}$ & $\begin{array}{l}\mu=0.07888 \\
(0.58,-497.4)\end{array}$ & $\begin{array}{l}C_{10}=8.57 \times 10^{-2} \\
C_{01}=-1.14 \times 10^{-1} \\
C_{20}=3.11 \times 10^{-5} \\
(0.07,-2479.7)\end{array}$ & $\begin{array}{l}C_{1}=1.00 \times 10^{-1} \\
C_{2}=6.42 \times 10^{-2} \\
C_{3}=8.08 \times 10^{-5} \\
(0.19,-1494.4)\end{array}$ & $\begin{array}{l}\mu_{1}=1.971 \times 10^{-19}, \alpha_{1}=18.341 \\
\mu_{2}=1.030, \alpha_{2}=2.729 \\
\mu_{3}=-1.059, \alpha_{3}=2.649 \\
(\mathbf{0 . 0 4}, \mathbf{- 2 9 8 8 . 9 )}\end{array}$ & $\begin{array}{l}C_{1}=43.77 \\
C_{2}=1.64 \times 10^{-1} \\
(0.15,-1699.2)\end{array}$ & $\begin{array}{l}C_{1}=31.76 \\
C_{2}=2.06 \times 10^{-3} \\
(0.02,-1443.8)\end{array}$ \\
\hline $\begin{array}{l}\text { Dragon Skin } 20 \\
\text { (S, AIC) }\end{array}$ & $\begin{array}{l}\mu=0.207047 \\
(1.28,197.4)\end{array}$ & $\begin{array}{l}C_{10}=2.47 \times 10^{-1} \\
C_{01}=-3.74 \times 10^{-1} \\
C_{20}=3.88 \times 10^{-5} \\
(0.11,-1764.2)\end{array}$ & $\begin{array}{l}C_{1}=1.00 \times 10^{-1} \\
C_{2}=1.69 \times 10^{-1} \\
C_{3}=2.66 \times 10^{-4} \\
(0.54,-493.8)\end{array}$ & $\begin{array}{l}\mu_{1}=-9.534 \times 10^{-1}, \alpha_{1}=3.478 \\
\mu_{2}=-1.4515, \alpha_{2}=3.181 \\
\mu_{3}=2.4085, \alpha_{3}=3.339 \\
(0.01,-3483.5)\end{array}$ & $\begin{array}{l}C_{1}=97.14 \\
C_{2}=1.964 \times 10^{-3} \\
(0.44,-645.8)\end{array}$ & $\begin{array}{l}C_{1}=67.45 \\
C_{2}=2.551 \times 10^{-3} \\
(0.57,-449.4)\end{array}$ \\
\hline $\begin{array}{l}\text { Dragon Skin } 30 \\
\text { (S, AIC) }\end{array}$ & $\begin{array}{l}\mu=0.24203 \\
(1.12,71.2)\end{array}$ & $\begin{array}{l}C_{10}=2.47 \times 10^{-1} \\
C_{01}=-3.30 \times 10^{-1} \\
C_{20}=2.09 \times 10^{-4} \\
(0.08,-1541.2)\end{array}$ & $\begin{array}{l}C_{1}=1.00 \times 10^{-1} \\
C_{2}=1.19 \times 10^{-1} \\
C_{3}=6.04 \times 10^{-4} \\
(0.36,-623.4)\end{array}$ & $\begin{array}{l}\mu_{1}=3.816 \times 10^{-2}, \alpha_{1}=3.417 \\
\mu_{2}=2.524 \times 10^{-2}, \alpha_{2}=3.417 \\
\mu_{3}=4.456 \times 10^{-2}, \alpha_{3}=3.417 \\
(0.17,-1084.5)\end{array}$ & $\begin{array}{l}C_{1}=57.05 \\
C_{2}=3.849 \times 10^{-3} \\
(0.29,-757.4)\end{array}$ & $\begin{array}{l}C_{1}=38.77 \\
C_{2}=4.987 \times 10^{-3} \\
(0.40,-565.4)\end{array}$ \\
\hline $\begin{array}{l}\text { Dragon Skin FX-Pro + Slacker } \\
\text { (S, AIC) }\end{array}$ & $\begin{array}{l}\mu=6.0145 \times 10^{-3} \\
(0.35,-1693.5)\end{array}$ & $\begin{array}{l}C_{10}=4.71 \times 10^{-3} \\
C_{01}=-1.79 \times 10^{-2} \\
C_{20}=4.73 \times 10^{-6} \\
(0.04,-5070.5)\end{array}$ & $\begin{array}{l}C_{1}=1.00 \times 10^{-1} \\
C_{2}=2.67 \times 10^{-3} \\
C_{3}=6.39 \times 10^{-6} \\
(0.06,-4365.6)\end{array}$ & $\begin{array}{l}\mu_{1}=6.159 \times 10^{-1}, \alpha_{1}=2.695 \\
\mu_{2}=-1.136, \alpha_{2}=2.791 \\
\mu_{3}=5.323 \times 10^{-1}, \alpha_{3}=2.876 \\
(0.02,-6073.5)\end{array}$ & $\begin{array}{l}C_{1}=1.727 \\
C_{2}=2.069 \times 10^{-3} \\
(0.02,-3831.1)\end{array}$ & $\begin{array}{l}C_{1}=1.574 \\
C_{2}=2.151 \times 10^{-3} \\
(0.09,-3749.3)\end{array}$ \\
\hline $\begin{array}{l}\text { Psycho Paint } \\
\text { (S, AIC) }\end{array}$ & $\begin{array}{l}\mu=0.15826 \\
(0.99,-5.9)\end{array}$ & $\begin{array}{l}C_{10}=1.65 \times 10^{-1} \\
C_{01}=-2.18 \times 10^{-1} \\
C_{20}=3.22 \times 10^{-4} \\
(0.08,-1507.0)\end{array}$ & $\begin{array}{l}C_{1}=1.00 \times 10^{-1} \\
C_{2}=1.07 \times 10^{-1} \\
C_{3}=6.00 \times 10^{-4} \\
(0.24,-858.9)\end{array}$ & $\begin{array}{l}\mu_{1}=-8.916 \times 10^{-2}, \alpha_{1}=5.048 \\
\mu_{2}=3.779 \times 10^{-2}, \alpha_{2}=3.884 \\
\mu_{3}=8.842 \times 10^{-2}, \alpha_{3}=5.047 \\
(\mathbf{0 . 0 3 1},-\mathbf{- 2 1 0 8 . 2})\end{array}$ & $\begin{array}{l}C_{1}=2.013 \times 10^{1} \\
C_{2}=6.435 \times 10^{-3} \\
(0.23,-890.1)\end{array}$ & $\begin{array}{l}C_{1}=14.94 \\
C_{2}=7.621 \times 10^{-3} \\
(0.29,-746.4)\end{array}$ \\
\hline $\begin{array}{l}\text { Mold Star } 16 \text { FAST } \\
(\mathrm{S}, \text { AIC) }\end{array}$ & $\begin{array}{l}\mu=0.14204 \\
(0.95,-33.8)\end{array}$ & $\begin{array}{l}C_{10}=1.84 \times 10^{-1} \\
C_{01}=-3.31 \times 10^{-1} \\
C_{20}=1.73 \times 10^{-5} \\
(0.3,-471.0)\end{array}$ & $\begin{array}{l}C_{1}=1.00 \times 10^{-1} \\
C_{2}=1.08 \times 10^{-1} \\
C_{3}=2.82 \times 10^{-4} \\
(0.44,-595.5)\end{array}$ & $\begin{array}{l}\mu_{1}=3.487 \times 10^{-2}, \alpha_{1}=5.048 \\
\mu_{2}=1.546 \times 10^{-2}, \alpha_{2}=3.884 \\
\mu_{3}=7.624 \times 10^{-3}, \alpha_{3}=5.047 \\
(0.27,-932.8)\end{array}$ & $\begin{array}{l}C_{1}=3.994 \times 10^{1} \\
C_{2}=3.148 \times 10^{-3} \\
(0.39,-670.5)\end{array}$ & $\begin{array}{l}C_{1}=28.66 \\
C_{2}=3.919 \times 10^{-3} \\
(0.47,-548.9)\end{array}$ \\
\hline $\begin{array}{l}\text { Mold Star 20T } \\
(\mathrm{S}, \mathrm{AIC})\end{array}$ & $\begin{array}{l}\mu=0.16984 \\
(1.08,55.9)\end{array}$ & $\begin{array}{l}C_{10}=2.20 \times 10^{-1} \\
C_{01}=-3.84 \times 10^{-1} \\
C_{20}=1.08 \times 10^{-5} \\
(0.2,1160.1)\end{array}$ & $\begin{array}{l}C_{1}=1.00 \times 10^{-1} \\
C_{2}=1.32 \times 10^{-1} \\
C_{3}=3.24 \times 10^{-4} \\
(0.52,-467.3)\end{array}$ & $\begin{array}{l}\mu_{1}=-1.734, \alpha_{1}=3.801 \\
\mu_{2}=3.857, \alpha_{2}=3.710 \\
\mu_{3}=-2.120, \alpha_{3}=3.609 \\
(0.13,-1439.3)\end{array}$ & $\begin{array}{l}C_{1}=51.149 \\
C_{2}=2.979 \times 10^{-3}\end{array}$ & $\begin{array}{l}C_{1}=36.086 \\
C_{2}=3.769 \times 10^{-3} \\
(0.55,-427.7)\end{array}$ \\
\hline $\begin{array}{l}\text { SOLOPLAST } 150318 \\
(\mathrm{~S}, \mathrm{AIC})\end{array}$ & $\begin{array}{l}\mu=0.12323 \\
(1.36,201.4)\end{array}$ & $\begin{array}{l}C_{10}=1.01 \times 10^{-1} \\
C_{01}=-1.68 \times 10^{-1} \\
C_{20}=4.91 \times 10^{-4} \\
(0.13,-1338.6)\end{array}$ & $\begin{array}{l}C_{1}=1.00 \times 10^{-1} \\
C_{2}=5.88 \times 10^{-2} \\
C_{3}=6.73 \times 10^{-4} \\
(0.22,-959.2)\end{array}$ & $\begin{array}{l}\mu_{1}=3.588 \times 10^{-1}, \alpha_{1}=3.515 \\
\mu_{2}=2.653 \times 10^{-1}, \alpha_{2}=3.515 \\
\mu_{3}=-6.199 \times 10^{-1}, \alpha_{3}=3.480 \\
(\mathbf{0 . 0 6},-\mathbf{- 1 7 2 8 . 0 )}\end{array}$ & $\begin{array}{l}C_{1}=7.958 \\
C_{2}=1.014 \times 10^{-2} \\
(0.30,-773.5)\end{array}$ & $\begin{array}{l}C_{1}=6.44 \\
C_{2}=1.111 \times 10^{-2} \\
(0.34,-689.0)\end{array}$ \\
\hline
\end{tabular}



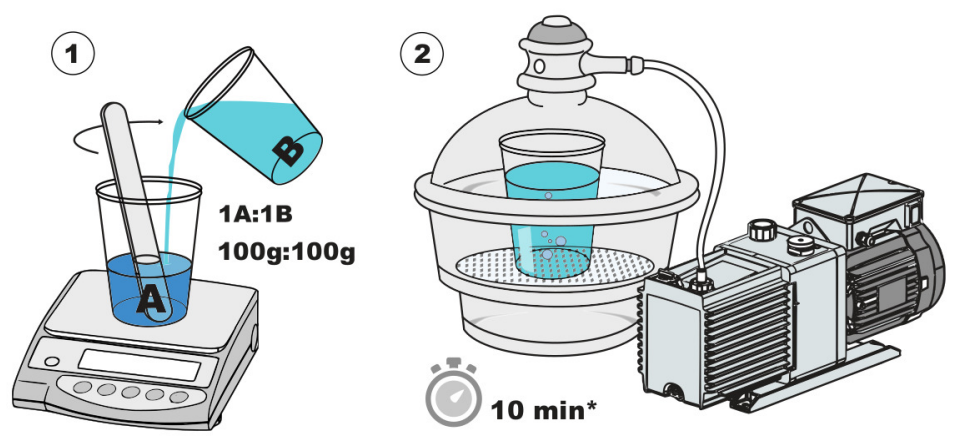

(4)

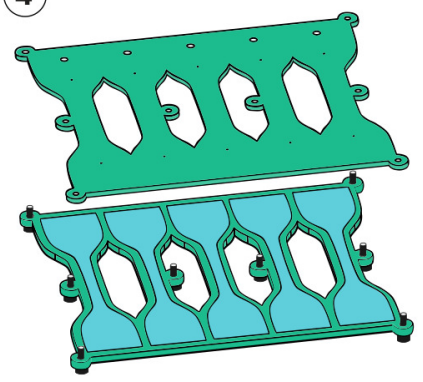

3

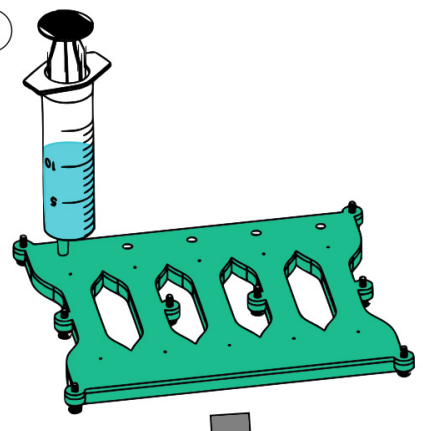

(5)

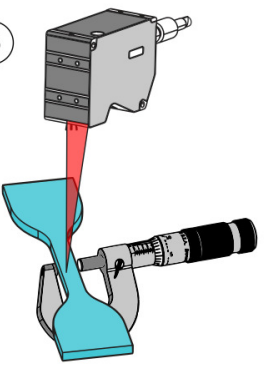

(6)

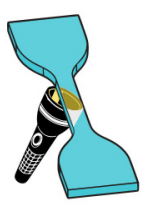

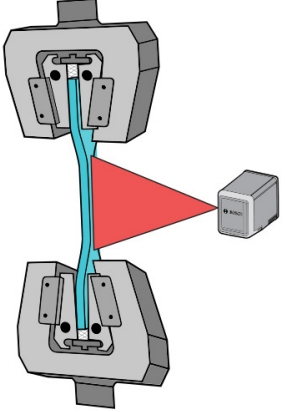

Fig. 1. Specimens fabrication steps: (1) Mixing of the two parts, (2) de-airing the mixture, (3) pouring into molds (4), curing for $24 \mathrm{~h}$, (5) measuring the dimensions, (6) inspecting the quality. *4 min for PlatSilGel-10, Mold Star 16 FAST and Body Double SILK

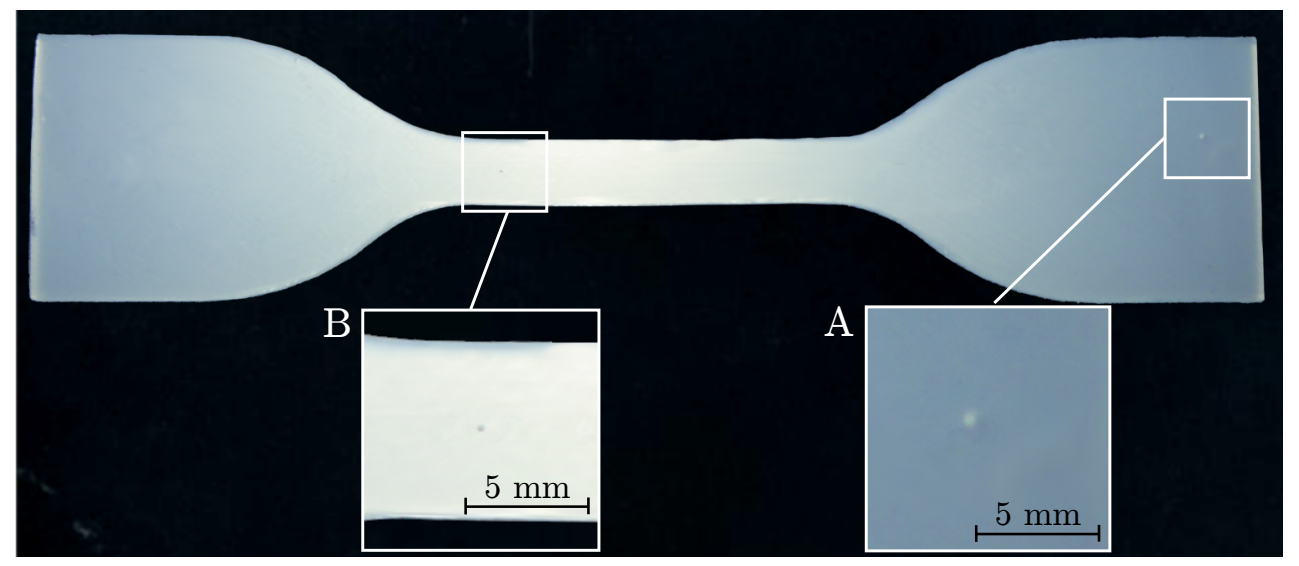

Fig. 2. Example of DragonSkin FX Pro specimen inspected with backlight. The presence of bubbles were only allowed in the shoulders in the region that are clamped in the grips as in (A) but this sample was discarded due to the presence of one bubble in the gauge-length (B). 


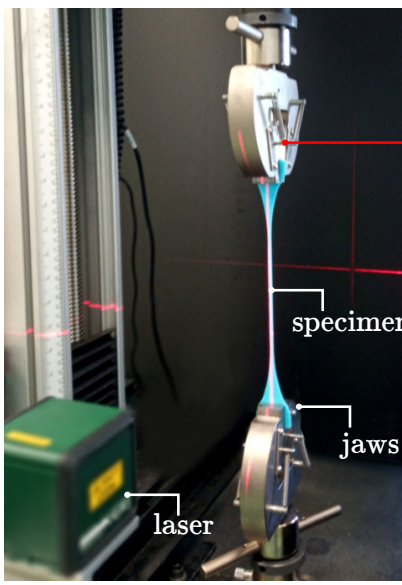

Fig. 3. Experimental setup for of the tensile test and details of the Instron mechanical wedge action grip of type 2710-010.

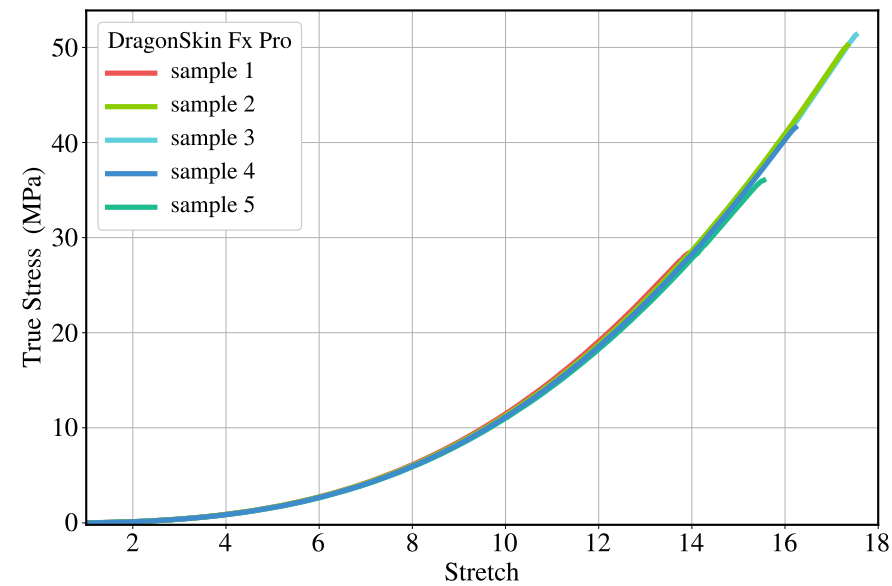

Fig. 4. Uniaxial tensile stress-stretch curves from five repeated tensile pull-to-failure tests on the Dragon Skin FX-Pro specimens at a strain rate of $450 \mathrm{~mm} / \mathrm{min}$. 


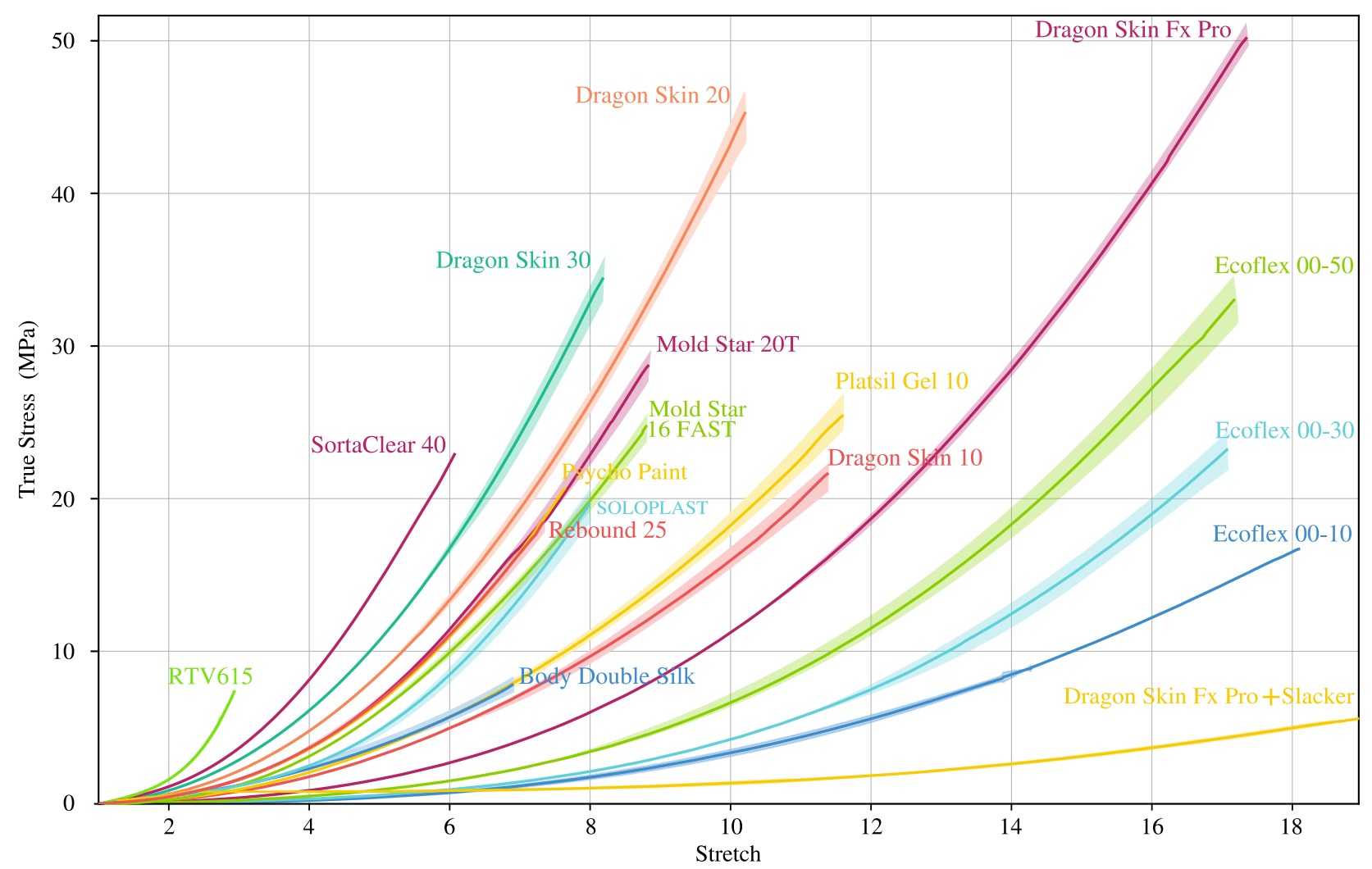

Fig. 5. Experimental uniaxial tensile stress-stretch pull to failure responses with $95 \%$ confidence bands.
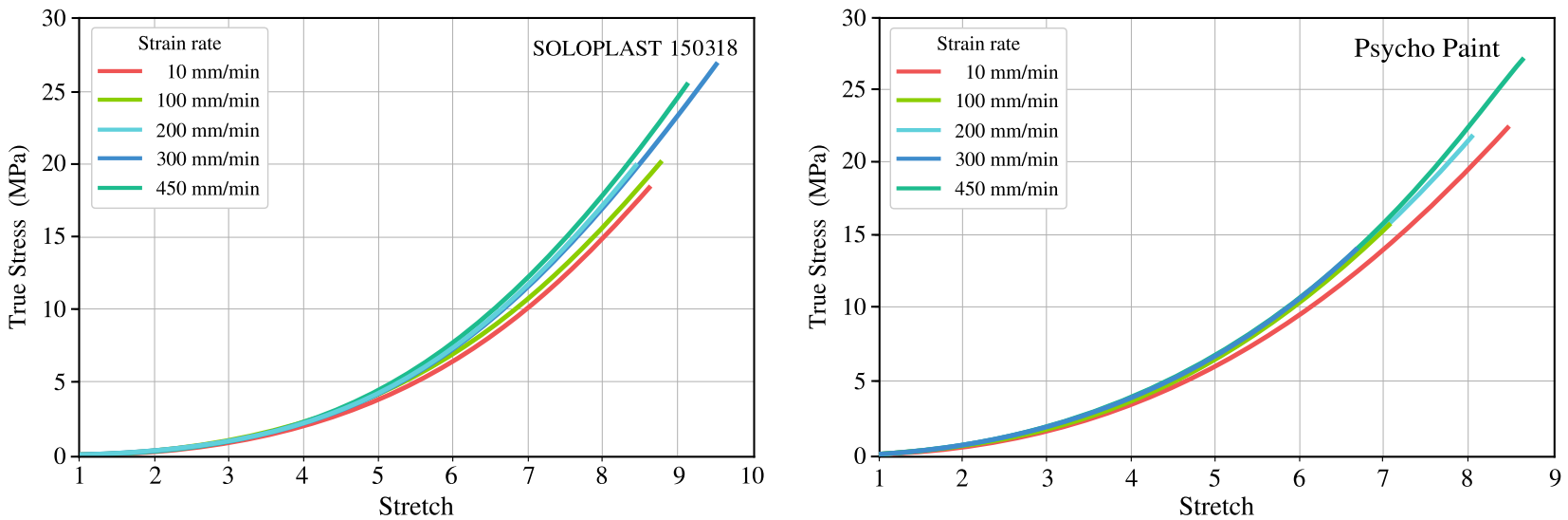

Fig. 6. Up to failure tensile test stress-stretch data, at five different strain rates, for SOLOPLAST 150318 and Psycho Paint. 

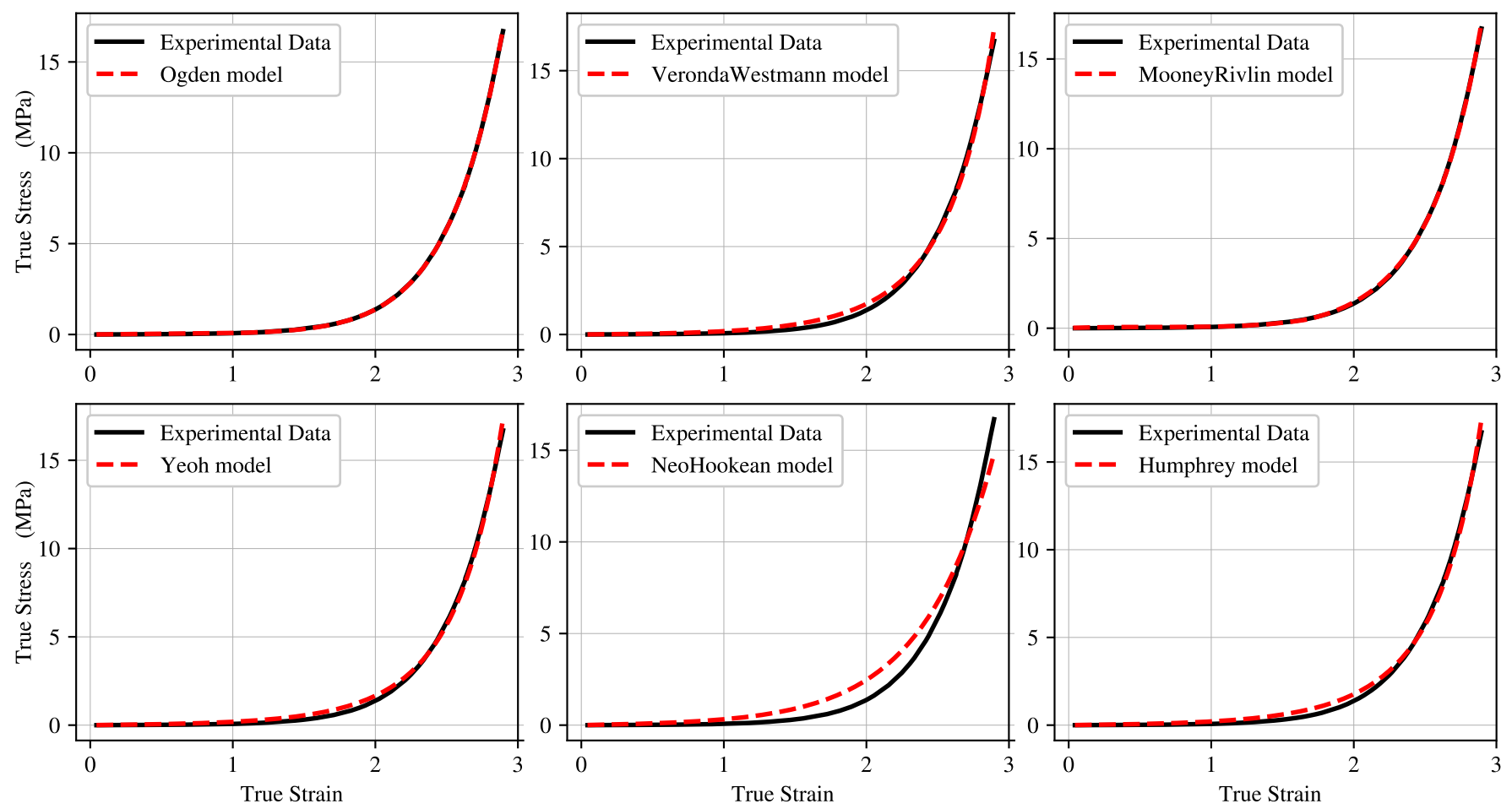

Fig. 7. Comparison between experimental data and predictions from least-squares fitting through Neo-Hookean, Mooney Rivlin, Yeoh, Ogden and VerondaWestmann models for the Ecoflex 00-10. 


\section{APPENDIX}

Example of the implemented Python code used to fit data with the Ogden model.

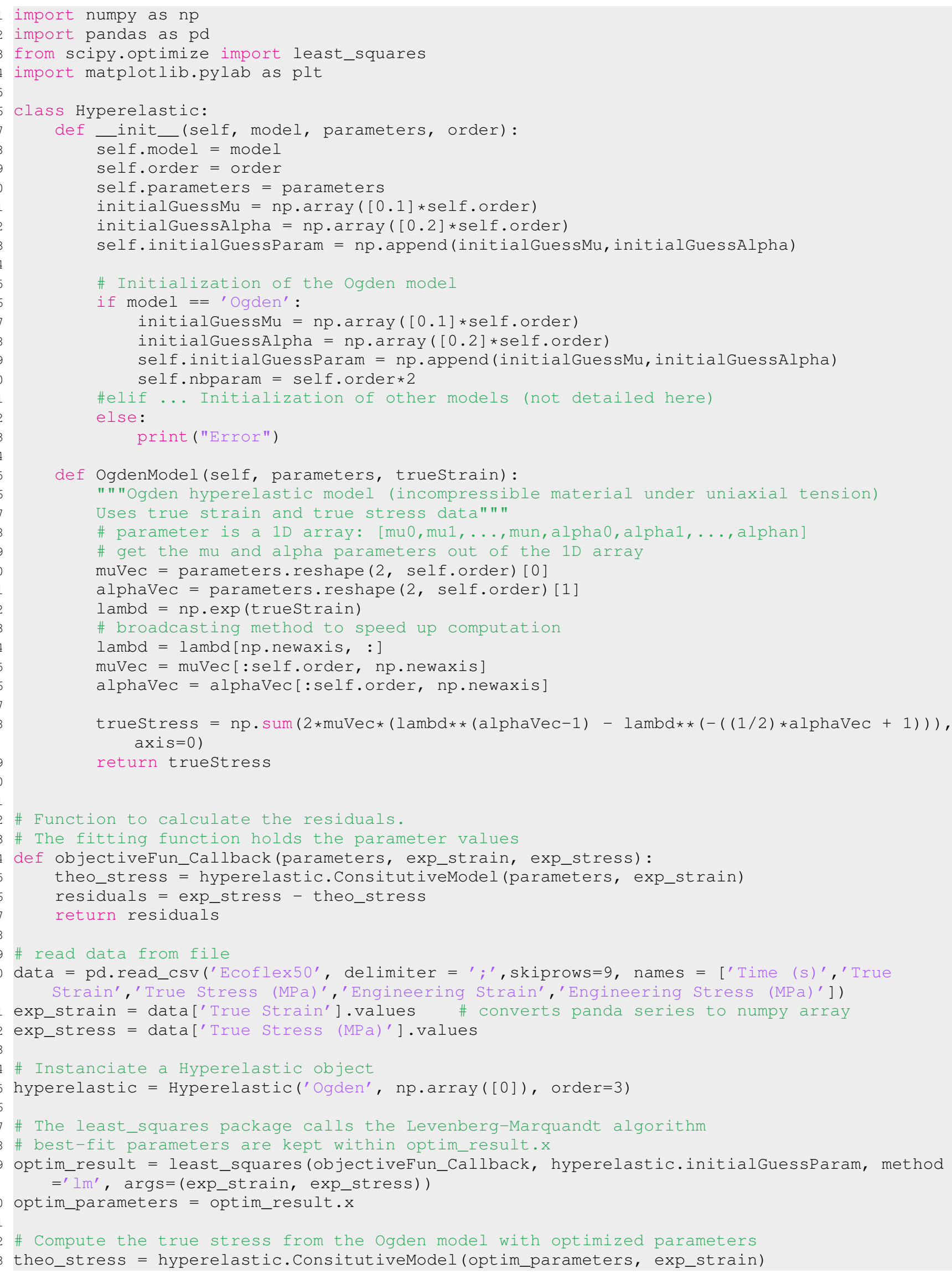


65 \# Plot experimental and predicted data on the same graph

66 plt.plot (exp_strain, exp_stress,' k', linewidth=2)

67 plt.plot (exp_strain, theo_stress,' - $^{--^{\prime}}$, linewidth=2) 